\title{
Ramanujan Type Congruences for a Partition Function
}

\author{
Haijian Zhao and Zheyuan Zhong
}

Center for Combinatorics, LPMC-TJKLC

Nankai University, Tianjin, P.R. China

haijian.nankai@gmail.com, zhongzheyuan@gmail.com

Submitted: Nov 8, 2010; Accepted: Mar 1, 2011; Published: Mar 11, 2011

Mathematics Subject Classifications: 05A17, 11P83

\begin{abstract}
We investigate the arithmetic properties of a certain function $b(n)$ given by $\sum_{n=0}^{\infty} b(n) q^{n}=(q ; q)_{\infty}^{-2}\left(q^{2} ; q^{2}\right)_{\infty}^{-2}$. One of our main results is $b(9 n+7) \equiv 0(\bmod 9)$
\end{abstract}

\section{Introduction}

Recently, Chan [5] introduced the function $a(n)$, which arised from his study of Ramanujan's cubic continued fraction. The function $a(n)$ is defined by

$$
\frac{1}{(q ; q)_{\infty}\left(q^{2} ; q^{2}\right)_{\infty}}=\sum_{n=0}^{\infty} a(n) q^{n} .
$$

Throughout this paper, we assume $|q|<1$ and we adopt the customary notation

$$
(a ; q)_{\infty}=\prod_{n=1}^{\infty}\left(1-a q^{n-1}\right) .
$$

There are many similar properties between $a(n)$ and the standard partition function $p(n)$, see $[5-9,11]$ for examples. One of the nice results of $a(n)$ is the generating function of $a(3 n+2)$ obtained by Chan [5], which states that

$$
\sum_{n=0}^{\infty} a(3 n+2) q^{n}=3 \frac{\left(q^{3} ; q^{3}\right)_{\infty}^{3}\left(q^{6} ; q^{6}\right)_{\infty}^{3}}{(q ; q)_{\infty}^{4}\left(q^{2} ; q^{2}\right)_{\infty}^{4}}
$$

This identity was also proven by Baruah and Ojah [1] using the 3-dissections for $\varphi(-q)^{-1}$ and $\psi(q)^{-1}$, and by Cao [4] applying the 3 -dissection for $(q ; q)_{\infty}\left(q^{2} ; q^{2}\right)_{\infty}$. We will give another proof based on identities of cubic theta functions in Section 2. 
Later, Kim [10] studied the following function $\bar{a}(n)$ counting the number of overcubic partitions of $n$,

$$
\sum_{n=0}^{\infty} \bar{a}(n) q^{n}=\frac{(-q ; q)_{\infty}\left(-q^{2} ; q^{2}\right)_{\infty}}{(q ; q)_{\infty}\left(q^{2} ; q^{2}\right)_{\infty}}
$$

In this paper, we are interested in the function $b(n)$ defined by

$$
\frac{1}{(q ; q)_{\infty}^{2}\left(q^{2} ; q^{2}\right)_{\infty}^{2}}=\sum_{n=0}^{\infty} b(n) q^{n}
$$

Our main aim is to present certain arithmetic properties for $b(n)$. In Section 3, we will prove the following Ramanujan type congruence modulo 9 , that is, for any $n \geq 0$,

$$
b(9 n+7) \equiv 0(\bmod 9) .
$$

We also establish two Ramanujan type congruences modulo 5 and 7 by using two classical identities, that is, for any $n \geq 0$,

$$
b(5 n+4) \equiv 0(\bmod 5),
$$

and

$$
b(7 n+2) \equiv b(7 n+3) \equiv b(7 n+4) \equiv b(7 n+6) \equiv 0(\bmod 7) .
$$

\section{Preliminaries}

In this section, we use cubic theta functions to obtain a 3-dissection of $(q ; q)_{\infty}^{-1}\left(q^{2} ; q^{2}\right)_{\infty}^{-1}$, which reproduces Chan's identity, and then give a number of facts that will be used in the next section.

Now, let us recall the definition of cubic theta functions $A(q), B(q), C(q)$ due to Borwein et al. [3], namely,

$$
\begin{aligned}
& A(q)=\sum_{m, n=-\infty}^{\infty} q^{m^{2}+m n+n^{2}} \\
& B(q)=\sum_{m, n=-\infty}^{\infty} \omega^{m-n} q^{m^{2}+m n+n^{2}}, \quad \omega=\exp (2 \pi i / 3), \\
& C(q)=\sum_{m, n=-\infty}^{\infty} q^{m^{2}+m n+n^{2}+m+n} .
\end{aligned}
$$

Borwein et al. [3] established the following relations which are useful to our proofs. 


\section{Lemma 2.1.}

$$
\begin{aligned}
A(q) & =A\left(q^{3}\right)+2 q C\left(q^{3}\right), \\
B(q) & =A\left(q^{3}\right)-q C\left(q^{3}\right), \\
C(q) & =3 \frac{\left(q^{3} ; q^{3}\right)_{\infty}^{3}}{(q ; q)_{\infty}}, \\
A(q) A\left(q^{2}\right) & =B(q) B\left(q^{2}\right)+q C(q) C\left(q^{2}\right) .
\end{aligned}
$$

We now derive the 3 -dissection for $(q ; q)_{\infty}^{-1}\left(q^{2} ; q^{2}\right)_{\infty}^{-1}$.

Theorem 2.1. We have

$$
\frac{1}{(q ; q)_{\infty}\left(q^{2} ; q^{2}\right)_{\infty}}=\frac{A\left(q^{6}\right)\left(q^{9} ; q^{9}\right)_{\infty}^{3}}{\left(q^{3} ; q^{3}\right)_{\infty}^{4}\left(q^{6} ; q^{6}\right)_{\infty}^{3}}+\frac{q A\left(q^{3}\right)\left(q^{18} ; q^{18}\right)_{\infty}^{3}}{\left(q^{3} ; q^{3}\right)_{\infty}^{3}\left(q^{6} ; q^{6}\right)_{\infty}^{4}}+\frac{3 q^{2}\left(q^{9} ; q^{9}\right)^{3}\left(q^{18} ; q^{18}\right)_{\infty}^{3}}{\left(q^{3} ; q^{3}\right)_{\infty}^{4}\left(q^{6} ; q^{6}\right)_{\infty}^{4}}
$$

Proof. By (2.3), we see that

$$
\frac{q}{(q ; q)_{\infty}\left(q^{2} ; q^{2}\right)_{\infty}}=\frac{q C(q) C\left(q^{2}\right)}{9\left(q^{3} ; q^{3}\right)_{\infty}^{3}\left(q^{6} ; q^{6}\right)_{\infty}^{3}}
$$

From (2.1), (2.2) and (2.4), we find that

$$
\begin{aligned}
q C(q) C\left(q^{2}\right) & =A(q) A\left(q^{2}\right)-B(q) B\left(q^{2}\right) \\
& =3 q C\left(q^{3}\right) A\left(q^{6}\right)+3 q^{2} A\left(q^{3}\right) C\left(q^{6}\right)+3 q^{3} C\left(q^{3}\right) C\left(q^{6}\right) .
\end{aligned}
$$

By combining (2.6) and (2.7) together, we obtain that

$$
\frac{q}{(q ; q)_{\infty}\left(q^{2} ; q^{2}\right)_{\infty}}=\frac{q C\left(q^{3}\right) A\left(q^{6}\right)+q^{2} A\left(q^{3}\right) C\left(q^{6}\right)+q^{3} C\left(q^{3}\right) C\left(q^{6}\right)}{3\left(q^{3} ; q^{3}\right)_{\infty}^{3}\left(q^{6} ; q^{6}\right)_{\infty}^{3}}
$$

which is equivalent to (2.5) upon using (2.3) to simplify it. This completes the proof.

From the above theorem, we immediately have the following corollary.

Corollary 2.1. Identity (1.1) holds, and

$$
\begin{aligned}
\sum_{n=0}^{\infty} a(3 n) q^{n} & =\frac{A\left(q^{2}\right)\left(q^{3} ; q^{3}\right)_{\infty}^{3}}{(q ; q)_{\infty}^{4}\left(q^{2} ; q^{2}\right)_{\infty}^{3}}, \\
\sum_{n=0}^{\infty} a(3 n+1) q^{n} & =\frac{A(q)\left(q^{6} ; q^{6}\right)_{\infty}^{3}}{(q ; q)_{\infty}^{3}\left(q^{2} ; q^{2}\right)_{\infty}^{4}} .
\end{aligned}
$$

Now, recall that Ramanujan theta functions $\varphi(q)$ and $\psi(q)$ which are defined as

$$
\begin{aligned}
& \varphi(q)=\sum_{n=-\infty}^{\infty} q^{n^{2}}, \\
& \psi(q)=\sum_{n=0}^{\infty} q^{n(n+1) / 2} .
\end{aligned}
$$

We need several properties of these two functions stated as the following lemmas. 


\section{Lemma 2.2.}

$$
\begin{aligned}
\varphi(-q) & =\frac{(q ; q)_{\infty}^{2}}{\left(q^{2} ; q^{2}\right)_{\infty}} \\
\psi(q) & =\frac{\left(q^{2} ; q^{2}\right)_{\infty}^{2}}{(q ; q)_{\infty}} .
\end{aligned}
$$

Proof. The above two identities are consequence of Jacobi's triple product identity. See [2, p.11] for the detail.

\section{Lemma 2.3.}

$$
\begin{aligned}
\psi(q) & =P\left(q^{3}\right)+q \psi\left(q^{9}\right), \\
\varphi(-q) & =\varphi\left(-q^{9}\right)-2 q Q\left(q^{3}\right),
\end{aligned}
$$

where

and

$$
P(q)=\frac{\left(q^{2} ; q^{2}\right)_{\infty}\left(q^{3} ; q^{3}\right)_{\infty}^{2}}{(q ; q)_{\infty}\left(q^{6} ; q^{6}\right)_{\infty}}
$$

$$
Q(q)=\frac{(q ; q)_{\infty}\left(q^{6} ; q^{6}\right)_{\infty}^{2}}{\left(q^{2} ; q^{2}\right)_{\infty}\left(q^{3} ; q^{3}\right)_{\infty}}
$$

Proof. With series manipulations, applying Jacobi's product identity, it is not hard to derive above identites and the detail is omitted here.

Lemma 2.4. If $r_{8}(n)$ and $t_{8}(n)$ are given by

$$
\begin{aligned}
& \varphi(q)^{8}=\sum_{n=1}^{\infty} r_{8}(n) q^{n}, \\
& \psi(q)^{8}=\sum_{n=0}^{\infty} t_{8}(n) q^{n} .
\end{aligned}
$$

Then

$$
\begin{aligned}
& r_{8}(n)=16(-1)^{n} \sum_{d \mid n}(-1)^{d} d^{3} \equiv(-1)^{n} \sum_{d \mid n}(-1)^{d} d(\bmod 3), \\
& t_{8}(n)=\sum_{\substack{d \mid n+1 \\
d \text { odd }}}\left(\frac{n+1}{d}\right)^{3} \equiv \sum_{\begin{array}{c}
d \mid n+1 \\
\text { o odd }
\end{array}} \frac{n+1}{d}(\bmod 3) .
\end{aligned}
$$

Proof. There are many proofs of the above facts, see, for example, [2, p.70, p.139].

Lemma 2.5. For any positive prime $p$,

$$
(q ; q)_{\infty}^{p} \equiv\left(q^{p} ; q^{p}\right)_{\infty}(\bmod p)
$$

Proof. The above fact is easily obtained by the following elementary fact

$$
(1-q)^{p} \equiv 1-q^{p}(\bmod p)
$$

and we omit the detail here.

With above lemmas, we can now move to the goal of proving the desired congruences. 


\section{Ramanujan Type Congruence Modulo 5,7 and 9}

In this section, we shall first use 3-dissection (2.5) to investigate the behavior of $b(3 n+1)$ modulo 9 which yields the desired congruence of $b(9 n+7)$. After that, we will apply Jacobi's identity to derive the congruence modulo 5 and use an identity of Ramanujan to establish the congruence modulo 7 .

Theorem 3.1. For any $n \geq 0$, we have

$$
b(9 n+7) \equiv 0(\bmod 9) .
$$

Note that the result in the above theorem is best possible, in the sense that the modulus, 3, cannot be replaced by a higher power of 3 .

Proof. By Theorem 2.1, we see that

$$
\sum_{n=0}^{\infty} b(n) q^{n}=\left(\frac{A\left(q^{6}\right)\left(q^{9} ; q^{9}\right)_{\infty}^{3}}{\left(q^{3} ; q^{3}\right)_{\infty}^{4}\left(q^{6} ; q^{6}\right)_{\infty}^{3}}+\frac{q A\left(q^{3}\right)\left(q^{18} ; q^{18}\right)_{\infty}^{3}}{\left(q^{3} ; q^{3}\right)_{\infty}^{3}\left(q^{6} ; q^{6}\right)_{\infty}^{4}}+\frac{3 q^{2}\left(q^{9} ; q^{9}\right)^{3}\left(q^{18} ; q^{18}\right)_{\infty}^{3}}{\left(q^{3} ; q^{3}\right)_{\infty}^{4}\left(q^{6} ; q^{6}\right)_{\infty}^{4}}\right)^{2}
$$

If we extract those terms from both sides of the above identity in which the power of $q$ is congruent to 1 modulo 3, we easily obtain that

$$
\sum_{n=0}^{\infty} b(3 n+1) q^{3 n+1} \equiv \frac{2 q A\left(q^{3}\right) A\left(q^{6}\right)\left(q^{9} ; q^{9}\right)_{\infty}^{3}\left(q^{18} ; q^{18}\right)_{\infty}^{3}}{\left(q^{3} ; q^{3}\right)_{\infty}^{7}\left(q^{6} ; q^{6}\right)_{\infty}^{7}}(\bmod 9)
$$

By dividing both sides of the above identity by $q$, replacing $q^{3}$ by $q$, we get

$$
\sum_{n=0}^{\infty} b(3 n+1) q^{n} \equiv \frac{2 A(q) A\left(q^{2}\right)\left(q^{3} ; q^{3}\right)_{\infty}^{3}\left(q^{6} ; q^{6}\right)_{\infty}^{3}}{(q ; q)_{\infty}^{7}\left(q^{2} ; q^{2}\right)_{\infty}^{7}}(\bmod 9)
$$

Now we need the following result

$$
\left(q^{3} ; q^{3}\right)_{\infty}^{3} \equiv(q ; q)_{\infty}^{9}(\bmod 9)
$$

which is obtained from the elementary fact

$$
(1-q)^{9} \equiv\left(1-q^{3}\right)^{3}(\bmod 9)
$$

By applying the above result in (3.2), we find that

$$
\sum_{n=0}^{\infty} b(3 n+1) q^{n} \equiv 2 A(q) A\left(q^{2}\right)(q ; q)_{\infty}^{2}\left(q^{2} ; q^{2}\right)_{\infty}^{2}(\bmod 9)
$$

By Lemma 2.2, we see that

$$
(q ; q)_{\infty}\left(q^{2} ; q^{2}\right)_{\infty}=\varphi(-q) \psi(q)
$$


By Lemma 2.3, we obtain

$$
\begin{aligned}
(q ; q)_{\infty}\left(q^{2} ; q^{2}\right)_{\infty} & =\left(P\left(q^{3}\right)+q \psi\left(q^{9}\right)\right)\left(\varphi\left(-q^{9}\right)-2 q Q\left(q^{3}\right)\right) \\
& =P\left(q^{3}\right) \varphi\left(-q^{9}\right)-q P\left(q^{3}\right) Q\left(q^{3}\right)-2 q^{2} Q\left(q^{3}\right) \psi\left(q^{9}\right) .
\end{aligned}
$$

Here the last equality is established by the fact that

$$
P(q) Q(q)=\left(q^{3} ; q^{3}\right)_{\infty}\left(q^{6} ; q^{6}\right)_{\infty}=\varphi\left(-q^{3}\right) \psi\left(q^{3}\right) .
$$

Now by (2.1), we have

$$
\begin{aligned}
A(q) A\left(q^{2}\right) & =\left(A\left(q^{3}\right)+2 q C\left(q^{3}\right)\right)\left(A\left(q^{6}\right)+2 q^{2} C\left(q^{6}\right)\right) \\
& \equiv A\left(q^{3}\right) A\left(q^{6}\right)+2 q A\left(q^{6}\right) C\left(q^{3}\right)+2 q^{2} A\left(q^{3}\right) C\left(q^{6}\right)(\bmod 9) .
\end{aligned}
$$

By substituting (3.4) and (3.5) into (3.3), we find that

$$
\begin{aligned}
\sum_{n=0}^{\infty} b(3 n+1) q^{n} \equiv & \left(A\left(q^{3}\right) A\left(q^{6}\right)+2 q A\left(q^{6}\right) C\left(q^{3}\right)+2 q^{2} A\left(q^{3}\right) C\left(q^{6}\right)\right) \times \\
& \left(P\left(q^{3}\right) \varphi\left(-q^{9}\right)-q P\left(q^{3}\right) Q\left(q^{3}\right)-2 q^{2} Q\left(q^{3}\right) \psi\left(q^{9}\right)\right)^{2}(\bmod 9) .
\end{aligned}
$$

Extracting those terms of form $q^{3 n+2}$, dividing by $q^{2}$ and replacing $q^{3}$ by $q$, yields that

$$
\begin{aligned}
\sum_{n=0}^{\infty} b(9 n+7) q^{n} & \equiv A(q) A\left(q^{2}\right)\left(P(q)^{2} Q(q)^{2}-4 P(q) Q(q) \varphi\left(-q^{3}\right) \psi\left(q^{3}\right)\right) \\
& +2 A(q) C\left(q^{2}\right)\left(P(q)^{2} \varphi\left(-q^{3}\right)^{2}+4 q P(q) Q(q)^{2} \psi\left(q^{3}\right)\right) \\
& +2 A\left(q^{2}\right) C(q)\left(-2 P(q)^{2} Q(q) \varphi\left(-q^{3}\right)+4 q Q(q)^{2} \psi\left(q^{3}\right)^{2}\right)(\bmod 9)
\end{aligned}
$$

Now noticing that $A(q) \equiv 1(\bmod 3)$, since

$$
A(q)=1+6 \sum_{n=0}^{\infty}\left\{\frac{q^{3 n+1}}{1-q^{3 n+1}}-\frac{q^{3 n+2}}{1-q^{3 n+2}}\right\},
$$

by [3] and $C(q) \equiv 0(\bmod 3)$ by $(2.3)$, using the relation that $P(q) Q(q)=\varphi\left(-q^{3}\right) \psi\left(q^{3}\right)$, we deduce that

$$
\begin{aligned}
\sum_{n=0}^{\infty} b(9 n+7) q^{n} & \equiv-3 P(q)^{2} Q(q)^{2}+2 C\left(q^{2}\right) P(q)^{2} \varphi\left(-q^{3}\right)^{2}+8 q C\left(q^{2}\right) P(q) Q(q)^{2} \psi\left(q^{3}\right) \\
& -4 C(q) P(q)^{2} Q(q) \varphi\left(-q^{3}\right)+8 q C(q) Q(q)^{2} \psi\left(q^{3}\right)^{2}(\bmod 9) .
\end{aligned}
$$

It is easy to check that

$$
C\left(q^{2}\right) P(q)^{2} \varphi\left(-q^{3}\right)^{2}=C(q) P(q)^{2} Q(q) \varphi\left(-q^{3}\right)=3 \frac{\left(q^{2} ; q^{2}\right)_{\infty}\left(q^{3} ; q^{3}\right)_{\infty}^{8}}{(q ; q)_{\infty}^{2}\left(q^{6} ; q^{6}\right)_{\infty}}
$$


and

$$
q C\left(q^{2}\right) P(q) Q(q)^{2} \psi\left(q^{3}\right)=q C(q) Q(q)^{2} \psi\left(q^{3}\right)^{2}=3 q \frac{(q ; q)_{\infty}\left(q^{6} ; q^{6}\right)_{\infty}^{8}}{\left(q^{2} ; q^{2}\right)_{\infty}^{2}\left(q^{3} ; q^{3}\right)_{\infty}} .
$$

Thus, to prove $b(9 n+7) \equiv 0(\bmod 9)$, it only needs to prove that

$$
-\left(q^{3} ; q^{3}\right)_{\infty}^{2}\left(q^{6} ; q^{6}\right)_{\infty}^{2}+\frac{\left(q^{2} ; q^{2}\right)_{\infty}\left(q^{3} ; q^{3}\right)_{\infty}^{8}}{(q ; q)_{\infty}^{2}\left(q^{6} ; q^{6}\right)_{\infty}}+q \frac{(q ; q)_{\infty}\left(q^{6} ; q^{6}\right)_{\infty}^{8}}{\left(q^{2} ; q^{2}\right)_{\infty}^{2}\left(q^{3} ; q^{3}\right)_{\infty}} \equiv 0(\bmod 3)
$$

which is equivalent to

$$
q \frac{\left(q^{2} ; q^{2}\right)_{\infty}^{16}}{(q ; q)_{\infty}^{8}}+\frac{(q ; q)_{\infty}^{16}}{\left(q^{2} ; q^{2}\right)_{\infty}^{8}} \equiv 1(\bmod 3)
$$

By the product formulae for $\varphi(-q)$ and $\psi(q)$, the above congruence can be rewritten as the following form

$$
q \psi(q)^{8}+\varphi(-q)^{8} \equiv 1(\bmod 3) .
$$

By the definitions of $r_{8}(n)$ and $t_{8}(n)$, one can show that the above identity is equivalent to

$$
(-1)^{n} r_{8}(n)+t_{8}(n-1) \equiv 0(\bmod 3)
$$

for all $n \geq 1$. This, in return, is equivalent to

$$
\sum_{d \mid n}(-1)^{d} d+\sum_{\substack{d \mid n \\ \text { dodd }}} \frac{n}{d} \equiv 0(\bmod 3)
$$

for all $n \geq 1$. To establish (3.6), let $n=2^{r} n_{1}$, where $\left(2, n_{1}\right)=1$. Then

$$
\begin{aligned}
\sum_{d \mid n}(-1)^{d} d+\sum_{\substack{d \mid n \\
\text { dodd }}} \frac{n}{d} & =\sum_{k=1}^{r} 2^{k} \sum_{d \mid n_{1}} d-\sum_{d \mid n_{1}} d+\sum_{d \mid n_{1}} \frac{2^{r} n_{1}}{d} \\
& =\left(\sum_{k=1}^{r} 2^{k}-1+2^{r}\right) \sum_{d \mid n_{1}} d \\
& =3\left(2^{r}-1\right) \sum_{d \mid n_{1}} d .
\end{aligned}
$$

This completes the congruence relation (3.6) and the proof is complete.

Now we turn to prove the following theorem.

Theorem 3.2. For any $n \geq 0$, we have

$$
b(5 n+4) \equiv 0(\bmod 5)
$$

and

$$
b(7 n+2) \equiv b(7 n+3) \equiv b(7 n+4) \equiv b(7 n+6) \equiv 0(\bmod 7) .
$$


Proof. By applying the case $p=5$ in Lemma 2.5, we obtain

$$
\sum_{n=0}^{\infty} b(n) q^{n} \equiv \frac{(q ; q)_{\infty}^{3}\left(q^{2} ; q^{2}\right)_{\infty}^{3}}{\left(q^{5} ; q^{5}\right)_{\infty}\left(q^{10} ; q^{10}\right)_{\infty}}(\bmod 5) .
$$

Thus, to prove that $b(5 n+4)$ is congruent to 0 modulo 5 , we only need to show that the coefficient of $q^{5 n+4}$ in the function $(q ; q)_{\infty}^{3}\left(q^{2} ; q^{2}\right)_{\infty}^{3}$ is a multiple of 5 . Using Jacobi's identity [2, p.14], namely,

$$
(q ; q)_{\infty}^{3}=\sum_{n=0}^{\infty}(-1)^{n}(2 n+1) q^{n(n+1) / 2}
$$

we have

$$
\begin{aligned}
(q ; q)_{\infty}^{3}\left(q^{2} ; q^{2}\right)_{\infty}^{3} & =\sum_{m=0}^{\infty}(-1)^{m}(2 m+1) q^{m(m+1) / 2} \sum_{n=0}^{\infty}(-1)^{n}(2 n+1) q^{n(n+1)} \\
& =\sum_{m=0}^{\infty} \sum_{n=0}^{\infty}(-1)^{m+n}(2 m+1)(2 n+1) q^{m(m+1) / 2+n(n+1)}
\end{aligned}
$$

If $m(m+1) / 2+n(n+1)$ is congruent to 4 modulo 5 , we must have $m \equiv n \equiv 2(\bmod 5)$, that is, $2 m+1$ and $2 n+1$ are both divided by 5 . This establishes the congruence (3.7).

Now we turn to the congruence modulo 7. By applying the case $p=7$ in Lemma 2.5, we get

$$
\begin{aligned}
\sum_{n=0}^{\infty} b(n) q^{n} & \equiv \frac{(q ; q)_{\infty}^{5}}{\left(q^{2} ; q^{2}\right)_{\infty}^{2}\left(q^{7} ; q^{7}\right)_{\infty}}(\bmod 7) \\
& =\frac{\varphi(-q)^{2}(q ; q)_{\infty}}{\left(q^{7} ; q^{7}\right)_{\infty}}
\end{aligned}
$$

By an identity of Ramanujan [2, p.20], namely,

$$
\varphi\left(-q^{2}\right)^{2}\left(q^{2} ; q^{2}\right)_{\infty}=\sum_{n=-\infty}^{\infty}(6 n+1) q^{3 n^{2}+n}
$$

we find that

$$
\sum_{n=0}^{\infty} b(n) q^{n} \equiv \frac{1}{\left(q^{7} ; q^{7}\right)_{\infty}} \sum_{n=-\infty}^{\infty}(6 n+1) q^{\left(3 n^{2}+n\right) / 2}(\bmod 7) .
$$

Since there are no integer $n$ with $\left(3 n^{2}+n\right) / 2$ congruent to 3,4 , or 6 modulo 7 , it follows that

$$
b(7 n+3) \equiv b(7 n+4) \equiv b(7 n+6) \equiv 0(\bmod 7) .
$$

If $\left(3 n^{2}+n\right) / 2 \equiv 2(\bmod 7)$ holds, then $n$ should be congruent to 1 modulo 7 , that is, $6 n+1$ is a multiple of 7 . This yields that $b(7 n+2) \equiv 0(\bmod 7)$, and we complete the proof. 


\section{References}

[1] N.D. Baruah and K.K. Ojah, Some congruences deducible from Ramanujan's cubic continued fraction, Int. J. Number Theory, to appear.

[2] B.C. Berndt, Number Theory in the Spirit of Ramanujan, Amer. Math. Soc., Providence, 2006.

[3] J.M. Borwein, P.B. Borwein and F.G. Garvan, Some cubic modular identites of Ramanujan, Trans. Amer. Math. Soc., 343 (1994), 35-47.

[4] Z. Cao, On Somos' dissection identities, J. Math. Anal. Appl., 365 (2010), 659-667.

[5] H.-C. Chan, Ramanujan's cubic continued fraction and a generalization of his "most beautiful identity", Int. J. Number Theory, 6 (2010), 673-680.

[6] H.-C. Chan, Ramanujan's cubic continued fraction and Ramanujan type congruences for a ceratin partition function, Int. J. Number Theory, 6 (2010), 819-834.

[7] H.-C. Chan, Distribution of a certain partition function modulo powers of primes, Acta Math. Sin., to appear.

[8] H.H. Chan and P.C. Toh, New analogues of Ramanujan's partition idenities, J. Number Theory, 130 (2010), 1898-1913.

[9] B. Kim, A crank analog on a certain kind of partition function arising from the cubic continued fraction, Acta Arith., to appear.

[10] B. Kim, The overcubic partition function mod 3, Proceedings of Ramanujan Rediscovered conference, to appear.

[11] J. Sinick, Ramanujan congruences for a class of eta quotients, Int. J. Number Theory, 6 (2010), 835-847. 